\title{
Thermoconvective Stability of a Ferrofluids in Presence of Magnetic Field
}

\author{
H. A. Jasmine* \\ Department of Mathematics, University of Rajshahi, Rajshahi, Bangladesh \\ Received 31 March 2016, accepted in final revised form 26 June 2016
}

\begin{abstract}
We have carried out a theoretical analysis of the linear thermoconvective stability of a ferrofluid, which is confined between two horizontal plates maintained at different constant temperatures and which is subject to an external uniform magnetic field in the vertical direction. The effects of the spin viscosity, vortex viscosity and magnetization relaxation are considered and discussed. The eigenvalue problem is solved by employing the Chebyshev pseudospectral method. It is found that the presence of magnetic field complements the buoyancy force in destabilizing the fluid at lower values of the magnetic field only and when the applied field is increased, the effect is reversed and the flow becomes more stable.
\end{abstract}

Keywords: Ferrofluids; Stability; Convection; Magnetic Field.

(C) 2016 JSR Publications. ISSN: 2070-0237 (Print); 2070-0245 (Online). All rights reserved. doi: http://dx.doi.org/10.3329/jsr.v8i3.27199

J. Sci. Res. 8 (3), 273-285 (2016)

\section{Introduction}

Ferrofluids or magnetic fluids are stable colloidal suspensions of fine ferromagnetic mono domain nanoparticles in a non-conducting carrier fluid. The particles are coated with a surfactant to avoid agglomeration and coagulation. Brownian motion, however, keeps the nanoparticles from settling under gravity. In the absence of applied field, the particles in a ferrofluid are randomly oriented and the fluid has no net magnetization. The fluid behaves similar to the carrier fluid. However, when placed in a strong magnetic field, these fluids flow toward regions of magnetic field and preserve their liquid character as long as the magnetic field is present. Recent investigations are also finding the use of ferrofluids in biomedical applications, such as drug targeting, as radio isotopes targeted by magnetic guidance and as a contrast agent for magnetic resonance imaging scans [1]. We remark that these fluids are different from the fluids

* Corresponding author: hosne_jasmine@ hotmail.com 
which are dispersions of micron sized magnetic particles, and in which the main interest of study appears to be related with non-Newtonian properties, very much like in polymeric fluids.

The continuum description of ferrofluids was initiated by Neuringer and Rosensweig [2]. This theory found several applications as described in the references cited above but assumed the collinearity of the magnetization with the magnetic field. This collinearity has been relaxed in several subsequent publications. First Shliomis [3] extended the theory by introducing the concepts of internal rotation of the particles and the magnetization relaxation equation. The general development of more sophisticated ferrofluid equations, which not only employs the concepts of internal rotation, magnetization relaxation, etc. but also introduces the role of asymmetric stress tensor. In some sense all these theories are generalizations of the ideas introduced earlier by Dahler and Scriven [4] and Condiff and Dahler [5] in the studies of structured continua and polarizable molecular fluids, respectively. Rosensweig [6] combined the effects of electrically conducting magnetic fluids with micropolar theory. Felderhof and Kroh [7], following the framework laid out in deGroot and Mazur [8], employed irreversible thermodynamics to arrive at the general set of equations. Rosensweig [9] has developed the governing equations derived on the basis of dynamic balance relationships and thermodynamic considerations. In this paper the author also demonstrates how the equations proposed in the earlier studies, Neuringer and Rosensweig [2] are related to the equations developed by him. We point out that there is quite a bit of similarity between the final form of equations proposed in Felderhof and Kroh [7] and Rosensweig [9]. In the present paper we have employed the general equations suggested in Rosensweig [6,10]. Some other aspects of the thermal convection problem in the above model have also been considered by Pérez et al. [11]. Ghofrani et al. [12] investigated experimentally on forced convection heat transfer of an aqueous ferrofluid flow passing through a circular copper tube in the presence of an alternating magnetic field. The effects of magnetic field, volume concentration, and Reynolds number on the convective heat transfer coefficient were widely examined leading to the determination of optimum conditions. Increase in the alternating magnetic field frequency and the volume fraction led to better heat transfer enhancement. The effect of the magnetic field in low Reynolds numbers was higher, and a maximum of $27.6 \%$ enhancement in the convection heat transfer was observed. Radha et al. [13] reported the experimental observation of diffraction patterns in a ferrofluid under the effect of magnetic field and gravity. The diffraction pattern showed a variation at different depths of the sample in the absence of the magnetic field and when the magnetic field is applied. The patterns also exhibited a change in the shape and size with variation of the external field. This effect arises due to thermally induced self-diffraction under the influence of gravity and external magnetic field.

To our knowledge, an attempt to study the convection problem in a vortex viscosity model using the equations proposed by Shliomis [3] was first made by 
Kaloni and Lou [14]. The work in this paper, which is applicable to dilute magnetic fluids, essentially discussed the convective stability results and compared them with the results obtained in Finlayson [15]. In the present paper we employ a general set of equations Rosensweig [6,10], which include vortex viscosity, $\xi$, magnetic relaxation, $\tau$, and spin viscosities, $\lambda, \eta$, to discuss the Rayleigh-Bénard problem, thus generalizing the results of Kaloni and Lou [14]. Our aim is to find the effect of the consideration and variation of these new quantities in the governing equations and also to compare our results, where possible, with the results of previous authors. It is to be noted that Zhan and Wainman [16] have employed these and the related equations in their studies leading to interesting results. We employ Chebyshev pseudospectral method to solve the eigenvalue problem and carry out calculations for Ester I base ferrofluids. Our calculations reveal that at sufficiently low values of $\alpha_{L}$, the Langevin parameter, the effect of consideration of $\xi, \tau, \lambda, \eta$ in the stability analysis is to accelerate the destabilization. At moderate to higher values of $\alpha_{L}$, this effect is reversed and as $\alpha_{L}$ increases the flow becomes more stable. In fact, at considerably higher values of $\alpha_{L}$ the Rayleigh number takes on values which are much higher than those for a viscous' fluid. Thus the effect of applied field is initially destabilizing but soon changes to stabilizing. Moreover, we find that the effect of increasing the vortex viscosity is to stabilize the flow while increasing magnetization relaxation time leads to destabilization of the flow. The effect of the spin viscosity when taken into account did not, however, show any change in the convection problem. In the case of gravity free environment, it is noted that the critical magnetic Rayleigh number, $R a_{m f, c}$, steadily increases from its lowest value at $\alpha_{L}=0$ to a value higher than when gravity is present, and then remains nearly constant, even at very high magnetic field.

The work presented is organized as follows. Section 2 discusses the general governing equations and then specialize these to the problem considered. The section 3 presents the numerical results and their discussion.

\section{Governing Equations}

The equations governing the flow of an incompressible ferrofluid

$$
\begin{aligned}
& \nabla \cdot \mathbf{q}=0, \\
& \rho_{0} \frac{\mathrm{Dq}}{\mathrm{Dt}}=-\nabla \mathrm{P}+(\mu+\xi) \nabla^{2} \mathbf{q}+\mu_{0}(\mathbf{M} \cdot \nabla) \mathbf{H}+2 \xi(\nabla \times \boldsymbol{\omega})-\rho \mathrm{g} \hat{\mathbf{k}}, \\
& \rho_{0} \mathrm{I} \frac{\mathrm{D} \boldsymbol{\omega}}{\mathrm{Dt}}=(\lambda+\eta) \nabla(\nabla \cdot \boldsymbol{\omega})+\eta \nabla^{2} \boldsymbol{\omega}+2 \xi(\nabla \times \mathbf{q}-2 \boldsymbol{\omega})+\mu_{0}(\mathbf{M} \times \mathbf{H}) \\
& \frac{\mathrm{DM}}{\mathrm{Dt}}=\boldsymbol{\omega} \times \mathbf{M}-\frac{1}{\tau}\left(\mathbf{M}-\mathbf{M}_{\mathrm{eq}}\right), \\
& {\left[\rho_{0} \mathrm{C}_{\mathrm{V}, \mathrm{H}}-\mu_{0} \mathbf{H} \cdot\left(\frac{\partial \mathbf{M}}{\partial \mathrm{T}}\right)_{\mathrm{V}, \mathrm{H}}\right] \frac{\mathrm{DT}}{\mathrm{Dt}}+\mu_{0} \mathrm{~T}\left(\frac{\partial \mathbf{M}}{\partial \mathrm{T}}\right)_{\mathrm{V}, \mathrm{H}} \cdot \frac{\mathrm{DH}}{\mathrm{Dt}}=\mathrm{K}_{\mathrm{T}} \nabla^{2} \mathrm{~T} .}
\end{aligned}
$$


The first three equations are the equations of mass balance, linear momentum and angular momentum, respectively. These are similar to the equations employed in polar fluid theories but are here augmented by the inclusion of the magnetic field. Equation (4) is the simpler form of the magnetization relaxation equation proposed by Shliomis [3]. Apart from some minor details, the formal structure of the magnetization equation in Shliomis [3], and Felderhof and Kroh [7] is nearly similar even though these have been derived differently.

Here $\boldsymbol{q}$ is the velocity, $\frac{\mathrm{D}}{\mathrm{Dt}}=\frac{\partial}{\partial \mathrm{t}}+\mathbf{q} \cdot \nabla, \rho_{0}$ is the mass density, $\mathrm{P}=\mathrm{p}+\frac{1}{2} \mu_{0} \mathrm{H}^{2}$ is the generalized pressure, $\omega$ is the average spin velocity of the colloidal particles, $\mu$ is the viscosity of the carrier fluid, $\xi$ is the vortex viscosity; and $\lambda, \eta$ are spin viscosities, $I$ is the average moment of inertia of the ferroparticle, $C_{V, H}$ is the heat capacity at constant volume, $k_{T}$ is the thermal conductivity, $T$ is the temperature, $\boldsymbol{H}$ is the magnetic field, $\boldsymbol{M}$ is the magnetization, $M_{e q}$ is the equilibrium magnetization, $\mu_{0}$ is the magnetic permeability (in free space $\mu_{0}=4 \pi \times 10^{-7} \mathrm{H} / \mathrm{m}$ ), $\boldsymbol{B}$ is the magnetic flux density and $\tau$ is the Brownian relaxation time.

Maxwell's equations in the magneto static limit are

$\nabla \cdot \mathbf{B}=0, \quad \nabla \times \mathbf{H}=\mathbf{0}, \quad \mathbf{B}=\mu_{0}(\mathbf{H}+\mathbf{M})$.

We also assume Boussinesq approximation for the density variation and write

$\rho g=\rho_{0} g\left[1-\alpha\left(T-T_{a}\right)\right]$,

where $\alpha$ is the thermal expansion coefficient and $T_{a}$ is the average temperature.

We assume that the equilibrium magnetization $M_{e q}$ is given by Langevin formula

$\mathbf{M}_{\mathrm{eq}}=\frac{\mathbf{H}}{\mathrm{H}} \mathrm{M}_{\mathrm{s}} \mathrm{L}\left(\alpha_{\mathrm{L}}\right)=\frac{\mathbf{H}}{\mathrm{H}} \mathrm{M}_{\mathrm{eq}}(\mathrm{H}, \mathrm{T}), \mathrm{L}\left(\alpha_{\mathrm{L}}\right)=\operatorname{coth}\left(\alpha_{\mathrm{L}}\right)-\frac{1}{\alpha_{\mathrm{L}}}, \alpha_{\mathrm{L}}=\frac{\mu_{0} \mathrm{mH}}{\mathrm{k}_{\mathrm{B}} \mathrm{T}}$,

where $M_{S}$ is the saturation magnetization, $m$ the magnetic moment of one particle and $k_{B}$ the Boltzmann constant. In the limit of low magnetic field $\left(\alpha_{L}<1\right)$, we define the initial susceptibility as

$\chi=\frac{\mu_{0} \mathrm{mM}_{\mathrm{s}}}{3 \mathrm{k}_{\mathrm{B}} \mathrm{T}_{\mathrm{a}}}=\frac{\mu_{0} \mathrm{~m}^{2} \mathrm{~N}}{3 \mathrm{k}_{\mathrm{B}} \mathrm{T}_{\mathrm{a}}}$,

where $N$ is the number of magnetic dipole per unit volume and $\mathrm{k}_{\mathrm{B}}=1.38 \times 10^{-23} \mathrm{~J} / \mathrm{K}^{-1}$.

We consider two horizontal plates of infinite extent which contain the ferrofluid and which are separated by a vertical distance $d$ apart. The temperatures of the upper and lower plates are maintained at $T_{1}$ and $T_{0}$ respectively. A uniform magnetic field is applied normal to the plates. The boundary conditions are:

$\mathbf{q}=\mathbf{0}, \boldsymbol{\omega}=\mathbf{0}$ on the rigid plates; $\mathrm{T}=\mathrm{T}_{0}$ at $\mathrm{z}=-\frac{\mathrm{d}}{2}, \mathrm{~T}=\mathrm{T}_{1}$ at $\mathrm{z}=\frac{\mathrm{d}}{2} ; \mathrm{T}_{\mathrm{a}}=\frac{1}{2}\left(\mathrm{~T}_{0}+\mathrm{T}_{1}\right)$ 
The magnetic boundary conditions are that the tangential component of the magnetic field and normal component of magnetic induction are continuous across the boundary.

In the quiescent state, following Finlayson [15], we express

$\mathrm{M}_{\mathrm{eq}}=\mathrm{M}_{\mathrm{a}}+\chi\left(\mathrm{H}-\mathrm{H}_{\mathrm{a}}\right)-\mathrm{K}_{1}\left(\mathrm{~T}-\mathrm{T}_{\mathrm{a}}\right)$,

where $\chi=\left(\frac{\partial M}{\partial H}\right)_{H_{a}, T_{a}}$ and $K_{1}=\left(\frac{\partial M}{\partial T}\right)_{H_{a}, T_{a}} \quad$ are susceptibility and pyromagnetic coefficients, respectively, where $\mathbf{M}=\left(\mathrm{M}_{\mathrm{x}}, \mathrm{M}_{\mathrm{y}}, \mathrm{M}_{\mathrm{z}}\right), \mathbf{H}=\left(\mathrm{H}_{\mathrm{x}}, \mathrm{H}_{\mathrm{y}}, \mathrm{H}_{\mathrm{z}}\right), \boldsymbol{\omega}=\left(\omega_{\mathrm{x}}, \omega_{\mathrm{y}}, \omega_{\mathrm{z}}\right)$ and. $\chi_{2}=\frac{M_{a}}{H_{a}}$ We have also used $\mathbf{H}=\nabla \phi$ since $\nabla \times \mathbf{H}=\mathbf{0}$.

The quiescent state solution of the basic equations (1)- (8) is

$$
\mathbf{q}_{\mathrm{b}}=0, \boldsymbol{\omega}_{\mathrm{b}}=0, \mathrm{~T}_{\mathrm{b}}=\mathrm{T}_{\mathrm{a}}-\beta \mathrm{z}, \beta=\frac{\mathrm{T}_{0}-\mathrm{T}_{1}}{\mathrm{~d}}, \mathbf{H}_{\mathrm{b}}=\left(\mathrm{H}_{\mathrm{a}}-\frac{\mathrm{K}_{1} \beta \mathrm{z}}{1+\chi}\right) \mathbf{k}, \mathbf{M}_{\mathrm{b}}=\left(\mathrm{M}_{\mathrm{a}}+\frac{\mathrm{K}_{1} \beta \mathrm{z}}{1+\chi}\right) \mathbf{k} .
$$

To study linear stability of the above solution we now perturb the system as

$$
\mathbf{q}=\mathbf{q}_{\mathrm{b}}+\mathbf{q}^{\prime}, \boldsymbol{\omega}=\boldsymbol{\omega}_{\mathrm{b}}+\boldsymbol{\omega}^{\prime}, \mathrm{T}=\mathrm{T}_{\mathrm{b}}+\theta, \mathbf{H}=\mathbf{H}_{\mathrm{b}}+\mathbf{H}^{\prime}, \mathbf{M}=\mathbf{M}_{\mathrm{b}}+\mathbf{M}^{\prime}, \mathrm{P}=\mathrm{P}_{\mathrm{b}}+\mathrm{P}^{\prime} .
$$

On introducing the following non-dimensional quantities

$$
\begin{aligned}
& (\mathrm{x}, \mathrm{y}, \mathrm{z})=\mathrm{d}\left(\mathrm{x}^{*}, \mathrm{y}^{*}, \mathrm{z}^{*}\right), \quad \mathrm{t}=\frac{\mathrm{d}^{2}}{\kappa} \mathrm{t}^{*}, \quad \mathrm{P}=\frac{\mu \kappa}{\mathrm{d}^{2}} \mathrm{P}^{*}, \quad \mathbf{q}=\frac{\kappa}{\mathrm{d}} \mathbf{q}^{*}, \quad \boldsymbol{\omega}=\frac{\kappa}{\mathrm{d}^{2}} \boldsymbol{\omega}^{*}, \quad \theta=\mathrm{d} \beta \theta^{*}, \\
& \mathbf{M}=\mathbf{M}_{\mathrm{a}} \mathbf{M}^{*}, \mathbf{H}=\mathrm{H}_{\mathrm{a}} \mathbf{H}^{*}, \kappa=\frac{\mathrm{K}_{\mathrm{T}}}{\rho_{0} \mathrm{C}_{\mathrm{V}, \mathrm{H}}}, \operatorname{Pr}=\frac{\nu}{\kappa}, \quad \mathrm{Ra}=\frac{\rho_{0} \mathrm{~g} \alpha \beta \mathrm{d}^{4}}{\mu \kappa}, \tau=\frac{\mathrm{d}^{2}}{\kappa} \tau^{*}, \quad \xi=\mu \xi_{1}, \\
& \mathrm{I}=\mathrm{d}^{2} \mathrm{I}_{1}, \eta=\mu \mathrm{d}^{2} \eta_{1} \\
& \lambda=\mu \mathrm{d}^{2} \lambda_{1}, \mathrm{M}_{1}=\frac{\mu_{0} \beta \chi^{2} \mathrm{H}_{\mathrm{a}}^{2}}{\rho_{0} \mathrm{~g} \alpha \mathrm{T}_{\mathrm{a}}^{2}(1+\chi)}, \mathrm{M}_{2}=\frac{\mu_{0} \chi^{2} \mathrm{H}_{\mathrm{a}}^{2}}{\rho_{0} \mathrm{C}_{\mathrm{V}, \mathrm{H}}(1+\chi) \mathrm{T}_{\mathrm{a}}}, \mathrm{M}_{3}=\frac{\mu_{0} \chi \mathrm{H}_{\mathrm{a}}^{2}}{\rho_{0} \mathrm{~g} \alpha \mathrm{dT}_{\mathrm{a}}}, \\
& \mathrm{Ra}_{\mathrm{m}}=\mathrm{Ra} \cdot \mathrm{M}_{1}=\frac{\mu_{0} \beta^{2} \chi^{2} \mathrm{H}_{\mathrm{a}}^{2} \mathrm{~d}^{4}}{\mu \kappa \mathrm{T}_{\mathrm{a}}^{2}(1+\chi)}, \mathrm{M}_{4}=\frac{(1+\chi) \mathrm{M}_{1}}{\chi \mathrm{M}_{3}}=\frac{\beta \mathrm{d}}{\mathrm{T}_{\mathrm{a}}},
\end{aligned}
$$

Where $\kappa$ is the fluid thermal diffusivity.

This gives the following set of non-dimensional linearized perturbation equations (dropping prime and asterisks)

$$
\begin{aligned}
& \nabla \cdot \mathbf{q}=0, \\
& \frac{1}{\operatorname{Pr}} \frac{\partial \mathbf{q}}{\partial \mathrm{t}}=-\nabla \mathrm{P}+\left(1+\xi_{1}\right) \nabla^{2} \mathbf{q}+\operatorname{Ra} \theta \hat{\mathbf{k}}+\mathrm{M}_{3} \operatorname{Ra}\left[\left(\frac{\chi_{2}}{\chi \mathrm{M}_{4}}+\frac{1}{1+\chi} \mathrm{z}\right) \frac{\partial \nabla \phi}{\partial \mathrm{z}}-\frac{\chi_{2}}{1+\chi} \mathrm{M}_{\mathrm{z}} \hat{\mathbf{k}}\right]+2 \xi_{1} \nabla \times \boldsymbol{\omega}, \\
& \frac{\mathrm{I}_{1}}{\operatorname{Pr}} \frac{\partial \boldsymbol{\omega}}{\partial \mathrm{t}}=\left(\lambda_{1}+\eta_{1}\right) \nabla(\nabla \cdot \boldsymbol{\omega})+\eta_{1} \nabla^{2} \boldsymbol{\omega}+2 \xi_{1}(\nabla \times \mathbf{q}-2 \boldsymbol{\omega})+\mathrm{M}_{3} \operatorname{Ra}\left(\frac{\chi_{2}}{\chi \mathrm{M}_{4}}+\frac{1}{1+\chi} \mathrm{z}\right)\left(-\frac{\partial \phi}{\partial \mathrm{y}} \hat{\mathbf{i}}+\frac{\partial \phi}{\partial \mathrm{x}} \hat{\mathbf{j}}\right)
\end{aligned}
$$




$$
\begin{gathered}
+\chi_{2} \mathrm{M}_{3} \mathrm{Ra}\left(\frac{1}{\chi \mathrm{M}_{4}}-\frac{1}{1+\chi} \mathrm{z}\right)\left(\mathrm{M}_{\mathrm{y}} \hat{\mathbf{i}}-\mathrm{M}_{\mathrm{x}} \hat{\mathbf{j}}\right) \\
\frac{\partial \mathbf{M}}{\partial \mathrm{t}}+\frac{\chi \mathrm{M}_{4}}{\chi_{2}(1+\chi)} \mathrm{w} \hat{\mathbf{k}}=\left(1+\frac{\chi \mathrm{M}_{4} \mathrm{z}}{\chi_{2}(1+\chi)}\right)\left(\omega_{\mathrm{y}} \hat{\mathbf{i}}-\omega_{\mathrm{x}} \hat{\mathbf{j}}\right)-\frac{1}{\tau}\left(\mathbf{M}-\frac{\partial \phi}{\partial \mathrm{x}} \hat{\mathbf{i}}-\frac{\partial \phi}{\partial \mathrm{y}} \hat{\mathbf{j}}-\frac{\chi}{\chi_{2}} \frac{\partial \phi}{\partial \mathrm{z}} \hat{\mathbf{k}}+\frac{\chi \mathrm{M}_{4}}{\chi_{2}} \theta \hat{\mathbf{k}}\right) \\
\frac{\partial \theta}{\partial \mathrm{t}}+\frac{(1+\chi) \mathrm{M}_{2}}{\chi}\left(\mathrm{z}-\frac{1}{\mathrm{M}_{4}}\right) \frac{\partial^{2} \phi}{\partial \mathrm{t} \partial \mathrm{z}}=\nabla^{2} \theta+\left(\mathrm{M}_{2} \mathrm{M}_{4} \mathrm{z}+1-\mathrm{M}_{2}\right) \mathrm{w} \\
\chi_{2} \nabla \cdot \mathbf{M}+\nabla^{2} \phi=0 .
\end{gathered}
$$

We further non-dimensionalize the magnetic potential $\phi$ and the magnetization vector $M_{z}$ as $\phi=\left(\frac{\chi}{1+\chi} \mathrm{M}_{4}\right) \phi^{\prime}, \mathrm{M}_{\mathrm{z}}=\left(\frac{\chi}{1+\chi} \frac{\mathrm{M}_{4}}{\chi_{2}}\right) \mathrm{M}_{\mathrm{z}}^{\prime}$, respectively and omit dashes.

On taking curl curl of equation (15) and curl of equation (16), the vertical component of resulting equations becomes, respectively, as

$$
\frac{1}{\operatorname{Pr}} \frac{\partial}{\partial \mathrm{t}} \nabla^{2} \mathrm{w}=\left(1+\xi_{1}\right) \nabla^{4} \mathrm{w}+\operatorname{Ra}_{1}^{2} \theta-\frac{\mathrm{Ra}_{\mathrm{m}}}{1+\chi} \frac{\partial}{\partial \mathrm{z}} \nabla_{1}^{2} \phi-\frac{\mathrm{Ra}_{\mathrm{m}}}{1+\chi} \nabla_{1}^{2} \mathrm{M}_{\mathrm{z}}+2 \xi_{1} \nabla^{2} \Gamma,
$$

$$
\begin{aligned}
\frac{\mathrm{I}_{1}}{\operatorname{Pr}} \frac{\partial \Gamma}{\partial \mathrm{t}}=-2 \xi_{1} \nabla_{1}^{2} \mathrm{w}-4 \xi_{1} \Gamma+\eta_{1} \nabla^{2} \Gamma+\mathrm{Ra}_{\mathrm{m}}\left(\frac{\chi_{2}}{\chi \mathrm{M}_{4}}+\frac{\mathrm{z}}{1+\chi}\right) \nabla_{1}^{2} \phi \\
+\operatorname{Ra}_{\mathrm{m}}\left(\frac{1}{\chi \mathrm{M}_{4}}-\frac{\mathrm{z}}{1+\chi}\right)\left(\nabla^{2} \phi+\frac{\partial \mathrm{M}_{\mathrm{z}}}{\partial \mathrm{z}}\right),
\end{aligned}
$$

where $\nabla_{1}^{2}=\left(\partial^{2} / \partial \mathrm{x}^{2}\right)+\left(\partial^{2} / \partial \mathrm{y}^{2}\right)$.

Taking the divergence of magnetization equation (17) and using equation (19), we obtain

$\frac{\partial}{\partial \mathrm{t}} \nabla^{2} \phi=\frac{\partial \mathrm{w}}{\partial \mathrm{z}}-\chi_{2}\left(\frac{1+\chi}{\chi \mathrm{M}_{4}}+\frac{\mathrm{z}}{\chi_{2}}\right) \Gamma-\frac{1}{\tau}\left[\left(1+\chi_{2}\right) \nabla_{1}^{2} \phi+(1+\chi) \frac{\partial^{2} \phi}{\partial \mathrm{z}^{2}}-(1+\chi) \frac{\partial \theta}{\partial \mathrm{z}}\right]$.

Taking vertical component of equation (23), we have

$\frac{\partial \mathrm{M}_{\mathrm{z}}}{\partial \mathrm{t}}=-\mathrm{w}-\frac{1}{\tau}\left[\mathrm{M}_{\mathrm{z}}-\chi \frac{\partial \phi}{\partial \mathrm{z}}+(1+\chi) \theta\right]$.

Equations (18), (20), (21), (22) and (23) are five equations for five variables $\mathrm{w}, \Gamma, \phi, \mathrm{M}_{\mathrm{z}}$ and $\theta$. In these equations, $\operatorname{Pr}$ is the Prandtl number, $\xi_{1}$ is nondimensionalized vortex viscosity, $R a$ is the viscous Rayleigh number, $\chi$ is the susceptibility, $\eta_{1}$ is the non-dimensionalized spin viscosity, $\tau$ is the nondimensionalized magnetic relaxation and $M_{4}=\beta d / T_{a}$. Thus, in comparison to the problem in quasistationary theory [15], we now have three more parameters: $\xi_{1}, \eta_{1}$ and $\tau$ which influence the stability problem. We note that if we set $\xi_{1}=0$ in (20) and $\tau=0$ in (22) and rearrange the terms we recover the equations studied in Finlayson [15]. There are, however, no counterparts to equation (21), the angular momentum equation, and the magnetization equation (23) in quasiequilibrium theory Neuringer and Rosensweig [2]. 
In order to match the domain of Chebyshev pseudospectral-QZ method, we reset the present domain from $\left[-\frac{1}{2}, \frac{1}{2}\right]$ to $[-1,1]$ with coordinate transformation from $\mathrm{z}$ to $2 \mathrm{z}$ in equations (18), (20)-(23).

We now perform the standard normal mode analysis and look for the solution of variables $\mathrm{w}, \Gamma, \phi, \mathrm{M}_{\mathrm{Z}}, \theta$ in the form

$$
\left[\mathrm{w}, \Gamma, \phi, \mathrm{M}_{\mathrm{z}}, \theta\right]=\left[\mathrm{w}(\mathrm{z}), \Gamma(\mathrm{z}), \phi(\mathrm{z}), \mathrm{M}_{\mathrm{z}}(\mathrm{z}), \theta(\mathrm{z})\right] \exp \left[\sigma \mathrm{t}+\mathrm{i}\left(\mathrm{k}_{\mathrm{x}} \mathrm{x}+\mathrm{k}_{\mathrm{y}} \mathrm{y}\right)\right] .
$$

On substituting (24) into equation (18), (20)-(23) in the new domain, we obtain

$$
\begin{aligned}
& \frac{\sigma}{\operatorname{Pr}}\left(4 \mathrm{D}^{2}-\mathrm{k}^{2}\right) \mathrm{w}(\mathrm{z})=\left(1+\xi_{1}\right)\left(16 \mathrm{D}^{4}-8 \mathrm{k}^{2} \mathrm{D}^{2}+\mathrm{k}^{4}\right) \mathrm{w}(\mathrm{z})+2 \xi_{1}\left(4 \mathrm{D}^{2}-\mathrm{k}^{2}\right) \Gamma(\mathrm{z}) \\
& -\mathrm{k}^{2} \mathrm{Ra} \theta(\mathrm{z})+\frac{\mathrm{Ra}_{\mathrm{m}}}{1+\chi} \mathrm{k}^{2} \mathrm{D} \phi(\mathrm{z})+\frac{\mathrm{Ra}_{\mathrm{m}}}{1+\chi} \mathrm{k}^{2} \mathrm{M}_{\mathrm{z}}(\mathrm{z}) \\
& \sigma \frac{\mathrm{I}_{1}}{\operatorname{Pr}} \Gamma(\mathrm{z})=-2 \xi_{1}\left(4 \mathrm{D}^{2}-\mathrm{k}^{2}\right) \mathrm{w}(\mathrm{z})+\eta_{1}\left(4 \mathrm{D}^{2}-\mathrm{k}^{2}\right) \Gamma(\mathrm{z})-\mathrm{k}^{2} \operatorname{Ra} \mathrm{m}\left(\frac{\chi_{2}}{\chi \mathrm{M}_{4}}+\frac{1}{2(1+\chi)} \mathrm{z}\right) \phi(\mathrm{z}) \\
& -4 \xi_{1} \Gamma(\mathrm{z})+\mathrm{Ra}_{\mathrm{m}}\left(\frac{1}{\chi \mathrm{M}_{4}}-\frac{1}{2(1+\chi)} \mathrm{z}\right)\left[\left(4 \mathrm{D}^{2}-\mathrm{k}^{2}\right) \phi(\mathrm{z})+2 \mathrm{DM}_{\mathrm{z}}(\mathrm{z})\right] \\
& \sigma\left(4 \mathrm{D}^{2}-\mathrm{k}^{2}\right) \phi(\mathrm{z})=2 \mathrm{Dw}(\mathrm{z})-\chi_{2}\left(\frac{1+\chi}{\chi \mathrm{M}_{4}}+\frac{\mathrm{z}}{2 \chi}\right) \Gamma(\mathrm{z})+\frac{2(1+\chi)}{\tau} \mathrm{D} \theta(\mathrm{z}) \\
& \sigma \mathrm{M}_{\mathrm{z}}(\mathrm{z})=\mathrm{w}(\mathrm{z})-\frac{1}{\tau} \mathrm{M}_{\mathrm{z}}(\mathrm{z})+\frac{2 \chi}{\tau} \mathrm{D} \phi(\mathrm{z})-\frac{1+\chi}{\tau} \theta(\mathrm{z}) \\
& \sigma \theta(\mathrm{z})+\sigma \frac{(1+\chi) \mathrm{M}_{2}}{\chi}\left(\mathrm{z}-\frac{2}{\mathrm{M}_{4}}\right) \mathrm{D} \phi(\mathrm{z})=\left(4 \mathrm{D}^{2}-\mathrm{k}^{2}\right) \theta(\mathrm{z})+\left(1-\mathrm{M}_{2}+\frac{1}{2} \mathrm{M}_{2} \mathrm{M}_{4} \mathrm{z}\right) \mathrm{w}(\mathrm{z})
\end{aligned}
$$

With the boundary conditions

$\mathrm{w}=0, \Gamma=0, \theta=0, \quad$ at $\mathrm{z}= \pm 1 ; \mathrm{M}_{\mathrm{z}}+2 \mathrm{D} \phi-\mathrm{k} \phi=0$, at $\mathrm{z}=-1 ; \mathrm{M}_{\mathrm{z}}+2 \mathrm{D} \phi+\mathrm{k} \phi=0$, at $\mathrm{z}=+1$;

and

$\mathrm{Dw}=0$, at $\mathrm{z}= \pm 1, \quad$ on rigid-rigid surface

$\mathrm{Dw}=0$, at $\mathrm{z}=-1, \mathrm{D}^{2} \mathrm{w}=0$, at $\mathrm{z}=+1$, on rigid-free surface

$\mathrm{D}^{2} \mathrm{~W}=0$, at $\mathrm{z}= \pm 1$, on free-free surface

\section{Numerical Results and Discussion}

We begin our discussion by noting that the solution of the eigenvalue problem (25)(29), together with the boundary conditions (30), depends upon a number of parameters. In order to carry out the numerical calculations, it is necessary to assign or estimate various parameters. In this respect, we have adopted values of some physical quantities from the Table 1 [10]. Since most of the calculations here are based upon the Ester I base fluid, we only report the values in this case: $T_{a}=298 \mathrm{~K}, M_{s}=15900$ 
$\mathrm{A} / \mathrm{m}, \rho_{0}=1150 \mathrm{~kg} / \mathrm{m}^{3}, \quad \mu=0.014 \mathrm{~kg} /(\mathrm{m} . \mathrm{s}), K_{T}=0.31 \mathrm{~kg} . \mathrm{m} /\left(\mathrm{K} . \mathrm{s}^{3}\right), \alpha=8.1 \times 10^{-4}, \chi=$ $0.4, \chi_{2}=0.35, \operatorname{Pr}=146.2, \kappa=10^{-7} \mathrm{~m}^{2} / \mathrm{sec}$. For the remaining parameters we have adopted the values discussed and used by Schumacher et al. [17], as $\mathrm{I}=7.5 \times 10^{-17} \mathrm{~m}^{2}$ and $\eta=2 \times 10^{-15} \mathrm{~kg} . \mathrm{m} . \mathrm{s}^{-1}$. Following earlier writers Sunil and Mahajan [18], we have set $M_{2}=0$ in our calculations.

In the following, $R a_{c}$ represents the critical Rayleigh number, $R a_{m, c}$ is the critical magnetic Rayleigh number and $R a_{m f, c}$ is the critical magnetic Rayleigh number in the gravity free environment.

Table 1. Critical temperature differences $\left(\Delta \mathrm{T}_{\mathrm{c}}\right)$ and critical Rayleigh ratios $\left(\frac{\mathrm{Ra}_{\mathrm{c}}}{1707.76}\right)$ with applied magnetic field $\left(\mathrm{H}_{\mathrm{a}}\right)$ for Ester I base ferrofluid layer width $\mathrm{d}=1 \mathrm{~mm}$.

\begin{tabular}{lll}
\hline Applied magnetic field $\mathrm{Ha}(\mathrm{A} / \mathrm{m})$ & $\Delta \mathrm{T}_{\mathrm{c}}(\mathrm{K})$ & Rayleigh ratio \\
\hline 13205.4 & 86.04 & 0.3946 \\
26410.8 & 53.43 & 0.2451 \\
39616.2 & 70.984 & 0.3256 \\
52821.6 & 88.413 & 0.4055 \\
\hline
\end{tabular}

We remark that we carried out calculations for $R a_{c}$ and $R a_{m, c}$ for different values $d$, for the Ester I base ferrofluid, but noticed that significant variation occurred only for $d$ $=1 \mathrm{~mm}$. Our all subsequent calculation in this section are thus for $d=1 \mathrm{~mm}$. Moreover, the values considered for $\xi_{1}$ and $\tau$ are dimensionless values throughout.

Before proceeding with the discussion of the results we wish to make two remarks. First, in our calculation, we do not observe the oscillatory instability behavior. In view of the remark presented in Ryskin and Pleiner [19] we could state that the specific experimental conditions, which are required to produce oscillating convective instability in ferrofluids, do not meet in the present case. One of the reasons may be that the value of the Lewis number is relatively small for an Ester I base fluid, i.e., the thermal diffusion time is much shorter than the mass diffusion time. The second remark is that we did not notice the effect of spin viscosity in our calculations.

Regarding the experimental results reported in Schwab et al. [20], it can be concluded that at $H=40.8 \mathrm{kA} / \mathrm{m}$, the convection occurs at $R a_{d} / R_{c}=0.45$ where $R_{c}=1708.76$.

As a result the heat flux in a ferrofluid, keeping similar temperature difference, is nearly doubled compared to the viscous fluid case. In Table 1 we have provided the critical temperature difference and the critical Rayleigh ratio, for Ester I base ferrofluids, at $d=1 \mathrm{~mm}$. Our values, in fact, are smaller than those in Schwab et al. [20]. The difference could be because of the different base ferrofluids considered in the two cases and also because our results are for $d=1 \mathrm{~mm}$, whereas the results reported in Schwab et al. [20] are for $d=3 \mathrm{~mm}$.

We also note that for lower values of the applied magnetic field there is a decrease both in $\Delta T_{c}$ and $R a_{c} / R_{c}$, but both the values increase as the magnetic field increases. This indicates that the non dilute ferrofluid considered here undergoes a structural change as the magnetic field is increased. 


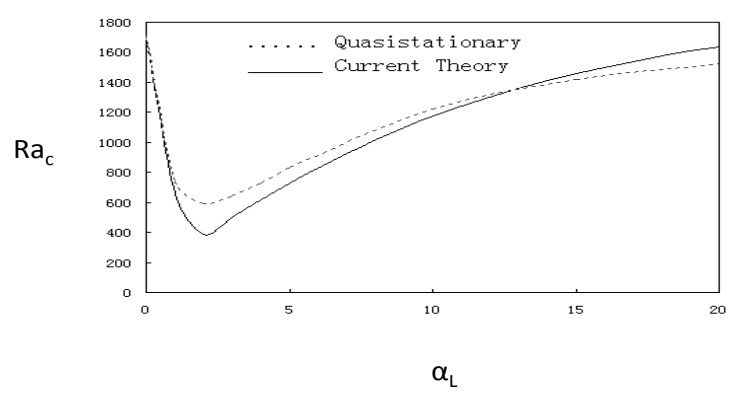

Fig. 1. Plot of the comparative values of the critical Rayleigh number $\mathrm{Ra}_{\mathrm{c}}$, between the qusistationary theory and the present theory for $\xi_{1}=0.2$, and $\tau=4.45 \times 10^{-4}$, and rigid-rigid boundaries.

Figs. 1 and 2 provide the comparative values of the critical Rayleigh number $R a_{c}$ and the critical magnetic Rayleigh number $R a_{m, c}$, between the qusistationary theory and the present theory. We carry out calculations for various other combination of the values of $\tau$ and $\xi_{1}$ and, eventhough there were some variations observed, consistent with the variations discussed subsequently, overall picture are similar to those as presented in Figs. 1 and 2. It is well known that an increase of temperature leads to the decrease in magnetization i.e. grad $T$ and $\operatorname{grad} M_{\mathrm{i}}$ act in the opposite direction. From equations (7) and (8), it also follows that $\operatorname{grad} \boldsymbol{M}$ and $\operatorname{grad} \boldsymbol{H}$ act in the opposite direction. Thus in the Rayliegh-Bénard problem, under consideration, in a magnetic fluid, where $\operatorname{grad} T$ is responsible for convection, the convection is accelerated because $\operatorname{grad} \boldsymbol{H}$ also acts in the same direction as grad $T$. As a result we have lower values for the critical Rayleigh number in a magnetic fluid. From these Figures, we also observed the tight coupling between the buoyancy and the magnetic forces. Whenever, there is the decrease of the Rayleigh number $R a_{c}$ (buoyancy related) there is corresponding increase in the magnetic Rayleigh number $R a_{m, c}$.

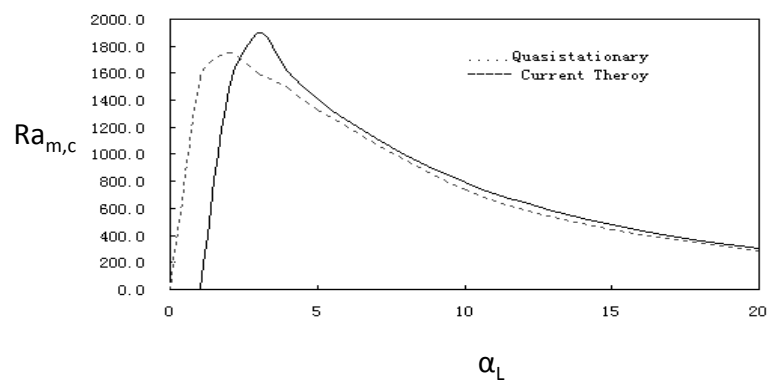

Fig. 2. The comparative values of the critical magnetic Rayleigh number $\operatorname{Ra}_{\mathrm{m}, \mathrm{c}}$, between the qusistationary theory and the present theory for $\xi_{1}=0.2$, and $\tau=4.45 \times 10^{-4}$, and rigid-rigid boundaries. 
When magnetization relaxation $\tau$ and vortex viscosity $\xi_{1}$ are considered, we have some interesting situations. First, the presence of a finite $\tau$ implies the involvement of the magnetic field for a longer period of time in the fluid (as compared to instantaneous relaxation or no relaxation in the quasistationary theory) and this adds up to the magnetic field already acting in the quasistationary theory. Accordingly, in the present case, we have a considerably greater value of grad $H_{\mathrm{i}}$ to add to grad $T$. As a result the convection is further accelerated and we have a lower value of $R a_{c}$ as compared to the quasistationary theory. This is clearly reflected in Figs. 1 and 2. With regard to the vortex viscosity we point out that it connects the linear momentum and the angular momentum equations and also relates the rate of transfer of internal to the external angular momentum. In the earlier studies, it was estimated to be $\xi_{1}=3 / 2 \mu \phi$ where $\phi$ is the hydrodynamic volume (fraction of the particles) and $\mu$ is the viscosity of the carrier fluid. The above expression, however, is valid only in a dilute regime.

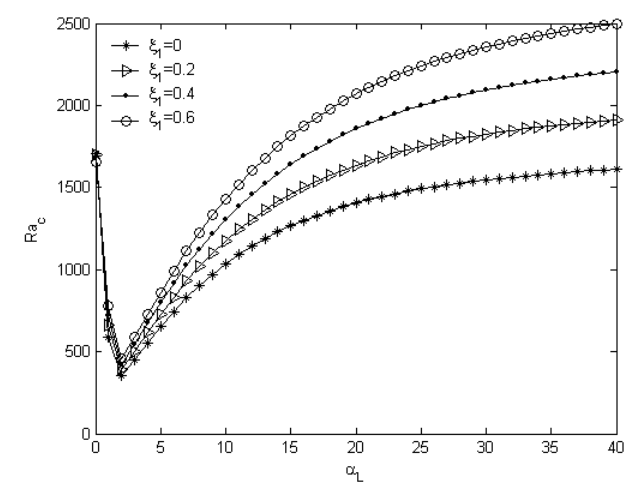

Fig. 3. Plot of $\mathrm{Ra}_{\mathrm{c}}$ (with gravity) with $\alpha_{\mathrm{L}}$ for Ester I base fluid and rigid-rigid boundaries. For different values of $\xi_{1}$, at $\tau=4.45 \times 10^{-2}$.

Now as the field increases, it is likely that the difference between the vorticity and spin decreases. This is so because in a non-dilute magnetic fluid, as the field is increased, the magnetic particle will start forming chains and thus offer more resistance to vorticity. Thus increasing field implies a steady increase in $\xi_{1}$. From Figs. 1 and 2, we notice that the effect of $\tau$ dominates for lower values of $\alpha_{L}$ and, when magnetic field is increased, is soon overtaken by the presence of $\xi_{1}$.

In order to have more clear idea of vortex viscosity variation, we have plotted the $R a_{c}$ against $\alpha_{L}$, for different values of $\xi_{1}$. Fig. 3 shows the variation of $R a_{c}$ at $\tau=4.45 \times 10^{-2}$. As can be seen from these Figs, we first note that, the general pattern remains the same for different values of $\xi_{1}$. That is, for low values of $\alpha_{L}, R a_{c}$ decreases, and when $\alpha_{L}$ values are increased further, reverse trend takes place. Now as $\alpha_{L}$ is increased, the values of $R a_{c}$ increase. For the specific variation of $\xi_{1}$, we find that, with the increase of $\alpha_{L}$, as $\xi_{1}$ increases so does $R a_{\mathrm{c}}$. The variation in $R a_{c}$ values, 
however, is significant at higher $\alpha_{L}$ values. We also carried out calculations for $\tau=1.12 \times 10^{-1}$. These, in general, showed a similar behavior except that for lower values of $\alpha_{L}, R a_{c}$ values were more prominent as compared to those at $\tau=4.45 \times 10^{-2}$. We thus can conclude that, an increase in the vortex viscosity leads to a more stabilized flow behavior. Fig. 4 shows the plot of $R a_{c}$ against $\alpha_{L}$ for different values of $\tau$, at $\xi_{1}=0.2$. Here again, the general pattern remains the same. $R a_{c}$ decrease up to certain value of $\alpha_{L}$, and then increases with the increases of $\alpha_{L}$. The variation of $\tau$, however, is significantly different from that of $\xi_{1}$. Now as $\tau$ increases the values of $R a_{c}$ decreases with $\alpha_{L}$. The flow is thus most stable at the lowest value of $\tau$. Thus unlike the effect of the variation of vortex viscosity, in which case the increase of vortex viscosity leads a more stabilized flow, the increase of magnetization $\tau$ leads to an unstable behavior.

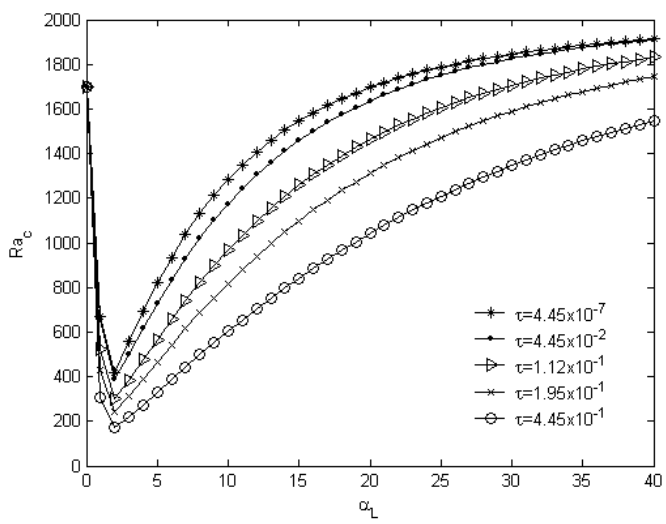

Fig. 4. Plot of $\mathrm{Ra}_{\mathrm{c}}$ (with gravity) with $\alpha_{\mathrm{L}}$ for Ester I base fluid and rigid-rigid boundaries. For different values of $\tau$ at $\xi_{1}=0.2$.

In Finlayson [15], to elaborate the tight coupling between the buoyancy and magnetic forces an equation of the type

$$
\frac{\mathrm{Ra}_{\mathrm{c}}}{1708}+\frac{\mathrm{Ra}_{\mathrm{m}, \mathrm{c}}}{2568}=1
$$

has been suggested. Our calculations reveal that this equation is nearly satisfied only at very low values of $\alpha_{\mathrm{L}}$. However, as $\alpha_{\mathrm{L}}$ increases, the first term in equation (31) alone takes on the values closer to 1 , or even greater than 1 in some cases, and thus this equation can not be satisfied. The tight coupling, as suggested in Finlayson [15], does not hold in the present case because of the increase of magneto viscous dissipation. 


\section{Conclusion}

In conclusion, we remark that the consideration of vortex viscosity, magnetization relaxation and spin viscosity, in the governing equations of the convection problem of a ferrofluid results in some typical behavior. We have found that application of a weak magnetic field promotes the convection. However, when the field strength is increased gradually, the convection process is slowed down and a further increase of the magnetic field, in fact, inhibits convection. We can thus conclude that in the convection problem in a magneticfluid, application of applied field is initially destabilizing before becoming stabilizing. We note that ferrofluid are structured fluids and contain a mixture of large and small particles. When the magnetic field is increased gradually, the larger size particles may form chains, and as these chains become larger, they could result in the increase of vortex viscosity.

To our knowledge this is the first study which has dealt with the convection problem in ferrofluids using a vortex viscosity model, with a specific magnetization relaxation equation. It will be interesting to study the convection problem in other model equations and to determine and compare the predictions based on different models.

\section{References}

1. Q. A. Pankhurst, J. Connolly, S. K. Jones, and J. Dobson, J. Phys. D: Appl. Phys. 36(13), R167 (2003). http://dx.doi.org/10.1088/0022-3727/36/13/201

2. J. L. Neuringer and R. E. Rosensweig, Phys. Fluids 7, 1927 (1964). http://dx.doi.org/10.1063/1.1711103

3. M. I. Shliomis, Sov. Phys. JETP 34, 1291 (1972).

4. J. S. Dahler, and L. E. Scriven, Proc. Roy. Soc. A 275, 504 (1963). http://dx.doi.org/10.1098/rspa.1963.0183

5. D. W. Condiff and J. S. Dahler, Phys. Fluids 7, 842 (1964). http://dx.doi.org/10.1063/1.1711295

6. R.E. Rosensweig, Science 271, 614 (1996). http://dx.doi.org/10.1126/science.271.5249.614

7. B.U. Felderhof and H. J. Kroh, J. Chem. Phys. 110, 7403(1999). http://dx.doi.org/10.1063/1.478642

8. S. R. deGroot and P. Mazur, Non-Equilibrium Thermodynamics (Dover, Mineola, New York, 1984).

9. R. E. Rosensweig, J. Chem, Phys. 121, 1228 (2004). http://dx.doi.org/10.1063/1.1755660

10. R. E .Rosensweig, Ferrohydrodynamics (Cambridge University Press, 1985).

11. L. M. Pérez, J. Bragard, D. Laroze, J. Martinez-Mardones, and H. Pleiner, J. Magn. Magn. Mat. 323, 691 (2011). http://dx.doi.org/10.1016/j.jmmm.2010.10.022

12. A. Ghofrani, M. H. Dibaei, A. H. Sima, and M. B. Shafii, J. Expthermflusci, 49, 193 (2013).

13. S. Radha, S. Mohan, and C. Pai, J. Phys. B 448, 341 (2014). http://dx.doi.org/10.1016/j.physb.2014.04.050

14. P. N. Kaloni and J. X. Lou, Phys. Rev. E 70, 026313 (2004). http://dx.doi.org/10.1103/PhysRevE.70.026313

15. B .A. Finlayson, J. Fluid Mech. 40, 753 (1970). http://dx.doi.org/10.1017/S0022112070000423 
16. M. Zhan and P. N. Wainman, J. Magn. Magn. Mat. 122, 323 (1993).

http://dx.doi.org/10.1016/0304-8853(93)91102-D

17. K. R. Schumacher, J. R. Riley, and B. A. Finlayson, J. Fluid Mech. 599, 1 (2008). http://dx.doi.org/10.1017/S0022112007009640

18. Sunil and A. Mahajan, Proc. Roy. Soc. A 464, 83 (2008).

http://dx.doi.org/10.1098/rspa.2007.1906

19. A. Ryskin and H. Pleiner, Phy. Rev. E 69, 046301. (2004). http://dx.doi.org/10.1103/PhysRevE.69.046301

20. L. Schwab, U. Hildebrandt, and K. Stierstadt, J. Magn. Magn. Mater. 39, 113 (1983). http://dx.doi.org/10.1016/0304-8853(83)90412-2 